\title{
Pandemie und Datenschutz
}

\section{Vielfältige Fragen aus aufsichtsbehördlicher Sicht}

\author{
Die Corona-Pandemie hat die Bedingungen für das Zusammenleben verändert. \\ Daraus ergeben sich auch Herausforderungen für den Datenschutz - ganz konkret \\ in zahlreichen Verarbeitungen, aber auch generell für Krisensituationen.
}

\section{Einleitung}

Im April 2020, zu einer Zeit, in der man noch nicht ahnte, wie lange die Pandemie andauern könnte, hat die Konferenz der unabhängigen Datenschutzaufsichtsbehörden (DSK) Datenschutz-

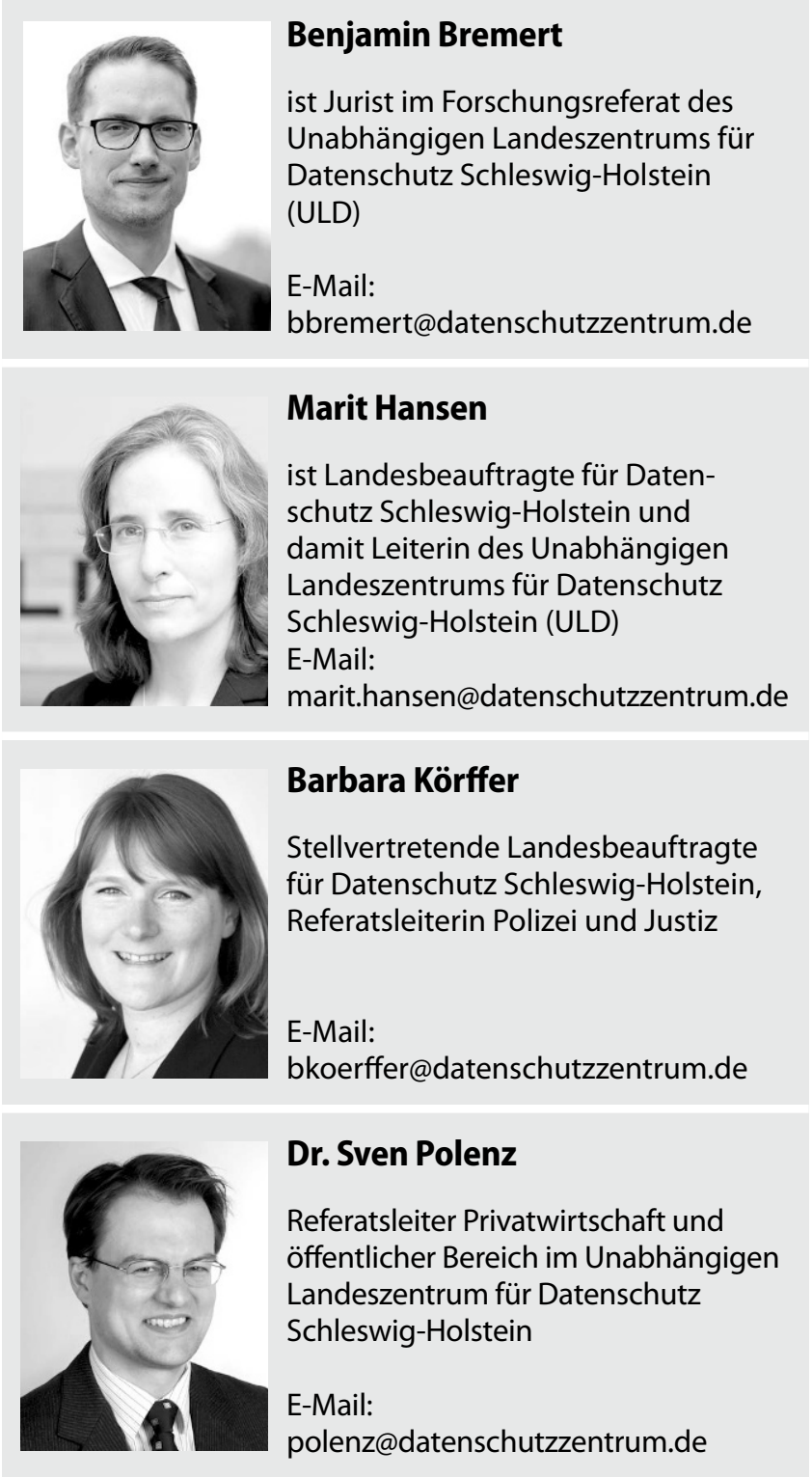

Grundsätze bei der Bewältigung der Corona-Pandemie beschlossen. ${ }^{1}$ Die DSK betont, dass auch in Krisenzeiten die DatenschutzGrundsätze gelten, und fordert geeignete Garantien zum Schutz der betroffenen Personen - das ist die ganze Bevölkerung - ein. Hier zeigen sich immer noch Defizite in rechtlichen Regelungen und in der Umsetzung in der Praxis.

Dies gilt besonders für Situationen, in denen Private (etwa Veranstalter oder Gastronomen) auf Grundlage einer Rechtspflicht zu einer Datenerhebung herangezogen werden und diese Daten, wie bei der Kontaktdatenerhebung, im Falle einer Infektion an die Behörden übermitteln müssen. Klar ist in diesen Fällen, dass die Privaten diese Daten nicht aus eigenem Interesse erheben, sondern aufgrund einer rechtlichen Verpflichtung tätig werden. Das etwaige Interesse, kein Bußgeld wegen der Zuwiderhandlung gegen die Datenerhebungspflicht auferlegt zu bekommen, ist dabei spiegelbildlich und akzessorisch zur Datenerhebungspflicht und ihrer Rechtsgrundlage zu sehen, stellt aber kein eigenes, isoliertes rechtliches Interesse dar. Gleiches gilt im Ergebnis unmittelbar für die Datenerhebung zu Zwecken der Infektionsbekämpfung: Ein Privater kann sich im Rahmen von Art. 6 Abs. 1 lit. $f$ DSGVO zwar auf die Wahrnehmung von Interessen Dritter berufen und sogar „bei Gelegenheit“ Allgemeininteressen fördern, er kann sich aber nicht zum Sachwalter öffentlicher Aufgaben heraufschwingen ${ }^{2}$, zumal das contra legem (Art. 6 Abs. 1 Satz 2 DSGVO) zu einer mittelbaren Anwendbarkeit für Fälle behördlicher Aufgabenerfüllung führen würde.

Dieser Text stellt eine Auswahl von Fragen der aufsichtsbehördlichen Praxis in Schleswig-Holstein vor: Verarbeitungen, die eine Infektion verhindern sollen (unten 2), Ansätze zum Nachverfolgen von Infektionsketten (3) sowie das Potenzial technischer Lösungen (4).

\section{Verhindern von Ansteckung}

\subsection{Datensammlung über Infizierte}

Die Gesundheitsämter verfügen über die Informationen zu positiven Testergebnissen bei Personen. Aus dem ganzen Bundesgebiet

\footnotetext{
* Die Erstellung dieses Aufsatzes erfolgte teilweise i. R. d. Forum Privatheit, das mit Mitteln des BMBF unter dem Förderkennzeichen 16KIS0747 (Forum Privatheit - Selbstbestimmtes Leben in der Digitalen Welt) gefördert wird.

1 DSK, Datenschutz-Grundsätze bei der Bewältigung der Corona-Pandemie, Entschließung vom 03.04.2020, https://www.datenschutzkonferenz-online.de/ media/en/Entschließung Pandemie 03_04_2020_final.pdf.

2 Robrahn/Bremert, Interessenskonflikte im Datenschutzrecht, ZD 2018, 291 (292).
} 
wurden Fälle bekannt, in denen auf diese Daten zugegriffen werden sollte. Das Argument angesichts einer Knappheit von Schutzausrüstung gegen Ansteckung: Sobald man wisse, dass bei einem Einsatz von Polizei oder Rettungsdiensten infizierte Personen betroffen seien, könne man mit besonderer Ausstattung und Hinweisen für den Schutz der eigenen Mitarbeitenden Sorge tragen.

Für das Land Schleswig-Holstein ist in einem solchen Fall das Gesundheitsdatenschutzgesetz (GDG SH) einschlägig. Nach $\$ 16$ Abs. 1 Nr. 3 GDG SH dürfen die Träger des Öffentlichen Gesundheitsdienstes diese Daten verarbeiten, soweit dies „erforderlich ist zur Abwehr einer Gefahr für Leben, Gesundheit oder Freiheit der betroffenen oder einer dritten Person und die Gefahr nicht auf andere Weise beseitigt werden kann". Dies betrifft auch eine Weitergabe der Daten: Eine standardmäßige Herausgabe von Listen wäre in den dargestellten Fällen nicht zulässig, sondern dies wäre allenfalls im Einzelfall und als letztes Mittel denkbar.

Insbesondere angesichts unerkannter Infektionen und des sich über die Zeit verändernden Ansteckungspotenzials positiv getesteter Personen ist jedoch die Aussagekraft der begehrten Daten ohnehin beschränkt. Damit wäre schon die Eignung einer Maßnahme wie der Weitergabe dieser Daten fraglich. Im Ergebnis muss man immer davon ausgehen, dass das Gegenüber ansteckend sein könnte.

\subsection{Symptomerhebung als Einlasskontrolle}

Vielfach sollten per Fragebogen Symptome der Covid-19-Erkrankung abgefragt werden, bevor eine Person Einlass bekam. Dies betraf in Schleswig-Holstein beispielsweise Gerichte und Schulen. Problematisch ist schon, dass einerseits die Covid-19-Symptome vielfältig sein können, andererseits aber kein einziges Symptom kennzeichnend für ein Ansteckungsrisiko ist, das sogar dann bestehen kann, wenn eine Person symptomlos ist. Viele Personen haben jedoch Allergien oder Atemwegserkrankungen, die nicht ansteckend sind und üblicherweise kein Abweisen am Eingang rechtfertigen. Für Anwältinnen und Anwälte bedeutete dies einen erheblichen Eingriff in ihre Berufsausübung. Schulpflichtige Kinder würden so vom Besuch des Unterrichts ausgeschlossen.

Bei den verwendeten Formularen waren die Rechtsgrundlagen für die Erhebung der Gesundheitsdaten nicht immer korrekt angegeben, die Informationen über die Verarbeitung der Daten und die Konsequenzen blieben oft unklar. Teilweise wurde nach Symptomen anderer Personen im selben Haushalt gefragt, ohne dass eine Rechtsgrundlage erkennbar war. Auch die Aufbewahrungsfristen schienen nicht immer durchdacht, z. B. eine Aussage, dass die Daten „acht Wochen nach Ende der Pandemie-Situation“ gelöscht werden sollen. Hier bestand Nachbesserungsbedarf.

\subsection{Temperaturmessung beim Zugang}

Würden Angaben zur Körpertemperatur von Besucherinnen und Besuchern oder Beschäftigten durch eine öffentliche Stelle erhoben, um mithilfe dieser Angaben den Zugang zu der Einrichtung zu steuern, so bedürfte es besonderer Rechtsgrundlagen. Da es sich bei der Körpertemperatur um ein Gesundheitsdatum handelt, muss Art. 9 Abs. 2 DSGVO beachtet werden. ${ }^{3}$ Einwilligungen (Artt. 9

3 DSK, Einsatz von Wärmebildkameras bzw. elektronischer Temperaturerfassung im Rahmen der Corona-Pandemie, Beschluss vom 10.09.2020, https://www. datenschutzkonferenz-online.de/media/dskb/20200910_beschluss_waeremebildkameras.pdf.
Abs. 2 Buchst. a, 7 DSGVO) erweisen sich dabei regelmäßig als ungeeignetes Instrument, da diese zu ihrer Wirksamkeit freiwillig erteilt werden müssen (vgl. auch Art. 6 Abs. 4 DSGVO). Besucherinnen und Besucher stehen vor der Entscheidung, entweder eine Messung zu dulden oder anderenfalls keinen Zugang zu der öffentlichen Einrichtung zu erhalten. Beschäftigte befinden sich zudem in einem Unterordnungsverhältnis zum Dienstherrn, was die Anforderungen an eine freiwillige Einwilligung erhöht. Messungen der Körpertemperatur führen auch nicht immer zu der Erkenntnis einer Infektion mit oder Erkrankung an Covid 19. So können fieberhafte Erkrankungen auch auf andere gesundheitliche Ursachen zurückgeführt werden oder z. B. mit der Schwangerschaft einer Person im Zusammenhang stehen. In jedem Fall wäre ein Besucher, der mit einem Messergebnis konfrontiert wird, veranlasst, nähere Erklärungen zu seinem Gesundheitszustand abzugeben, über deren Plausibilität dann wiederum nichtärztliches Personal entscheiden soll. Die Erklärungen führen dann auch zur Offenbarung weiterer Gesundheitsdaten, da beim Besucher der Eindruck erweckt wird, sich rechtfertigen zu müssen. Hierdurch würden zusätzliche Eingriffe in die Persönlichkeitsrechte des Besuchers erfolgen. Aber auch ein negatives Ergebnis wäre kein sicherer Anhaltspunkt dafür, ob eine Person nicht ansteckend ist, da eine Infektion mit SARS-CoV-2 im Durchschnitt erst einige Tage nach Eintritt der Infektiosität (präsymptomatisches Stadium) Symptome (und somit ggf. Fieber) entwickelt.

Beabsichtigt die öffentliche Stelle als datenschutzrechtlich Verantwortliche, die Messung der Körpertemperatur am Eingang zu einer öffentlichen Einrichtung als Service für Besucherinnen und Besucher bereitzustellen, indem Messungen freiwillig, durch die Personen selbst, unbeobachtet und ohne Speicherung des Messergebnisses erfolgen, so kann dies datenschutzrechtlich betrachtet zulässig sein.

Von Bedeutung wird aber sein, dass die öffentliche Stelle eine Erhebung von Gesundheitsdaten initiiert. Daher müssten für die Besucherinnen und Besucher zumindest die Pflichtinformationen nach Art. 13 DSGVO in einem Aushang bereitgestellt werden. Insbesondere wäre dort auf die Nichtvornahme einer Speicherung hinzuweisen sowie auf die Rechtsgrundlage (Einwilligung) der Erhebung der Gesundheitsdaten. Bezüglich der Einwilligung wären deren Vorgaben zu wahren, inkl. der Hinweise auf die Freiwilligkeit der Datenerhebung.

\subsection{Ampelsystem auf Basis von WLAN-Tracking}

In Betracht gezogen wird teilweise die Messung von Besucherströmen und der Personendichte in Freizeiteinrichtungen, um die ordnungsbehördliche Tätigkeit im Zusammenhang mit der Bekämpfung der Corona-Pandemie zu erleichtern und Besucherinnen und Besucher mit einem Ampelsystem auf mögliche Gefahren hinweisen. Man beabsichtigt, zusätzlich Schilder anzubringen, um auf die Erhebung der virtuellen dynamischen MACAdressen hinzuweisen und dadurch zu ermöglichen, dass die Besucherinnen und Besucher ihre WLAN-Signal-Empfangsbereitschaft in den mitgeführten Endgeräten abschalten.

Die Smartphones der Besucherinnen und Besucher senden demnach sog. virtuelle dynamische MAC-Adressen, die anders als die „echten“ für das Gerät eindeutigen, statischen MAC-Adressen ständig wechseln. Das WLAN der Gemeinde speichert nur die empfangenen virtuellen Adressen für ca. 30 Sekunden und löscht diese dann. Zurück bleibt eine fortlaufende Nummer. Begibt sich 
ein Besucher wieder in den Empfangsbereich des WLAN, wird erneut eine virtuelle dynamische MAC-Adresse erfasst und wieder eine fortlaufende Nummer vergeben. Es wird nicht die Angabe erfasst, dass es sich um dasselbe Smartphone handelt, zumal ein Abgleich mangels Speicherung nicht möglich wäre. Die fortlaufenden Nummern dienen der Zählung, wie viele Personen den Erfassungsbereich durchschreiten, um pandemiebedingt ggf. ordnungsbehördlich auf eine zu hohe Besucheranzahl reagieren zu können.

Bei den virtuellen dynamischen MAC-Adressen handelt es sich um personenbezogene Daten nach Art. 4 Nr. 1 DSGVO. Eine Zuordnung wäre möglich, auch wenn dies durch die nur kurzzeitige Speicherung technischen Aufwand erfordern würde.

Die pandemiebedingte Erfassung durch eine öffentliche Stelle als Verantwortlicher könnte ggf. auf Art. 6 Abs. 1 Buchst. e DSGVO gestützt werden. Demnach ist die Verarbeitung personenbezogener Daten zulässig, soweit diese für die Wahrnehmung einer Aufgabe erforderlich ist, die im öffentlichen Interesse liegt oder in Ausübung öffentlicher Gewalt erfolgt. Dabei kann es sich um die Wahrnehmung von ordnungsbehördlichen Aufgaben der Gefahrenabwehr handeln. Die Arbeit der Ordnungsbehörden soll nach Vorstellung der verantwortlichen Gemeinde erleichtert werden, indem Brennpunkte für eine hohe Besucherdichte auf öffentlichen Plätzen (Eingangsbereiche zu öffentlichen Einrichtungen, Marktplätze) identifiziert werden, um dann Abstandsregeln und andere Verpflichtungen kontrollieren zu können. Die Rechtsgrundlage für die kurzzeitige Erhebung der virtuellen dynamischen MAC-Adressen ist daher im Gefahrenabwehrrecht der Ordnungsbehörden zu suchen.

Ausgehend davon, dass die Befugnis zur Kontrolle der Abstandsregeln durch die datenschutzrechtlich verantwortliche Gemeinde besteht, ist die Bekämpfung der Corona-Pandemie ein zulässiger Zweck bzw. eine Aufgabe im öffentlichen Interesse nach Art. 6 Abs. 1 Buchst. e DSGVO. Die Verfolgung wirtschaftlicher oder touristischer Zwecke wäre in diesem Kontext unzulässig. Vor diesem Hintergrund könnte eine Rechtsgrundlage für die kurzeitige Verarbeitung der virtuellen dynamischen MACAdressen gesehen werden.

Von Bedeutung ist dabei, dass den Besucherinnen und Besuchern durch Hinweisschilder die Deaktivierung einer WLANEmpfangsbereitschaft ermöglicht wird. Weiterhin sind in diesem Kontext die Pflichtinformationen nach Art. 13 Abs. 1 und 2 DSGVO zu erfüllen. Dies kann etwa durch Aushänge erfolgen.

Mit dem etwaigen künftigen Wegfall einer ordnungsbehördlichen Kontrollbedürftigkeit (nach der erfolgreichen Bekämpfung der Corona-Pandemie) darf das System der Erfassung virtueller dynamischer MAC-Adressen nicht mehr genutzt werden, da der zulässige Verarbeitungszweck dann nicht mehr bestünde.

\subsection{Impfpass}

Ein umstrittener ${ }^{4}$ Ansatz, der immer wieder diskutiert ${ }^{5}$ wird, ist die Dokumentation einer SARS-CoV-2-Immunität nach einer durch-

4 Deutscher Ethikrat rät derzeit von Covid-19-Immunitätsbescheinigung ab, PM vom 22.09.2020, https://www.ethikrat.org/mitteilungen/2020/

deutscher-ethikrat-raet-derzeit-von-covid-19-immunitaetsbescheinigungen-ab/.

5 Kabinettsbeschluss: Mehr Tests und Corona-Immunitätsdokumentation im Impfpass, DAZ.online vom 29.04.2020, https://www.deutsche-apotheker-zeitung.de/news/artikel/2020/04/29/mehr-tests-und-immunitaetsdokumentationim-impfpass/chapter:all; Corona-App und Impfpass: Auf dem Weg in die ZweiKlassen-Gesellschaft, Berliner Zeitung vom 09.05.2020, https://www.

berliner-zeitung.de/zukunft-technologie/corona-app-und-impfpass-auf-dem- gestandenen Covid-19-Erkrankung. Dieser Ansatz ist fraglich, da Wissenschaftler gerade bei milden Infektionen davon ausgehen, dass die Antikörper innerhalb kürzester Zeit abgebaut werden und damit keine verlässliche Immunität bestünde. ${ }^{6}$ Außerdem droht bei Lockerungen von Corona-Maßnahmen für Personen mit dokumentierter Immunität die Gefahr, dass sich Personen vorsätzlich einer womöglich lebensgefährlichen Infektion aussetzen, um in den Genuss dieser Lockerungen zu kommen. Der andere Ansatz wäre die Dokumentation einer erfolgten Impfung, sobald ein entsprechender Impfstoff zur Verfügung steht. Diese Dokumentation wäre insoweit problemlos, als sie wie jede andere Impfung nach $\$ 22$ IfSG lediglich in den Impfpass bzw. Impfausweis eingetragen wird und dadurch eine schlichte Nachvollziehbarkeit von erfolgten Schutzimpfungen erreicht werden soll ${ }^{7}$. Problematisch würde es dann, wenn der entsprechende Eintrag im Impfpass zur Voraussetzung der Teilnahme am gesellschaftlichen Leben wird. Solchen generellen Überprüfungen dürfte jedenfalls ohne eine entsprechende Impfpflicht die Rechtsgrundlage fehlen.

\section{Nachverfolgen von Infektionsketten}

In vielen Bundesländern hat die Regierung Verordnungen mit Regelungen zu einem Hygienekonzept und zur Erhebung von Kontaktdaten erlassen, die von Gastronomen und Veranstaltern zu befolgen sind. In Schleswig-Holstein ist dies die Corona-Bekämpfungsverordnung ${ }^{8}$, die jeweils nach wenigen Wochen Geltung geändert und an die aktuelle Pandemie-Situation angepasst wurde. Eine der Änderungen betraf beispielsweise die Aufbewahrungszeiten für die erhobenen Kontaktdaten: Nachdem die Dauer in Schleswig-Holstein zunächst auf sechs Wochen festgelegt war, passte man die Verordnung später an die Zeiten in den benachbarten Bundesländern auf vier Wochen an.

\subsection{Kontaktdatenerhebung}

In $₫ 4$ Abs. 2 Corona-Bekämpfungsverordnung wird insbesondere geregelt: „Soweit nach dieser Verordnung Kontaktdaten erhoben werden, sind Erhebungsdatum und-uhrzeit, Vor-und Nachname, Anschrift, sowie, soweit vorhanden, Telefonnummer oder E-MailAdresse für einen Zeitraum von vier Wochen aufzubewahren und dann zu vernichten. Sie sind auf Verlangen der zuständigen Behörde zu übermitteln, sofern dies zum Zwecke der Nachverfolgung von möglichen Infektionswegen erforderlich ist. Es ist zu gewährleisten, dass unbefugte Dritte von den erhobenen Daten keine Kenntnis erlangen. Eine anderweitige Verwendung ist unzulässig. [...]“ Die zuständige Behörde ist das Gesundheitsamt, das im konkreten Infektionsfall die Aufgabe der Kontaktnachverfolgung hat.

Es wird - mittlerweile unter Bußgeldandrohung - verlangt, dass die Daten korrekt sind - denn sonst würde im Infektionsfall das Gesundheitsamt auf Basis falscher Daten arbeiten und zu-

weg-in-die-zwei-klassen-gesellschaft-li.83155; Debatte um Covid-19-Immunität: Ein Ausweis mit Risiken, tagesschau.de vom 22.09.2020, https://www.tagesschau. de/inland/immunitaetsausweis-ethikrat-101.html.

6 Ibarrondo et al., Rapid Decay of Anti-SARS-CoV-2 Antibodies in Persons with Mild Covid-19, The New England Journal of Medicine, DOI: 10.1056/NEJMc2025179 = https://www.nejm.org/doi/full/10.1056/nejmc2025179.

7 Vgl. die knappe Gesetzesbegründung zur Vorgängerregelung in § 16 Bundes-Seuchengesetz (BT-Drs. 3/1888, S. 23).

8 https://www.schleswig-holstein.de/DE/Schwerpunkte/Coronavirus/Erlasse/Landesverordnung_Corona.html. 
mindest Ressourcen vergeuden. Allerdings sind diejenigen, die ihre Kontaktdaten hergeben müssen, nicht verpflichtet, ihren Personalausweis gegenüber den Gastronomen oder Veranstaltern zu zeigen, $\$ 20$ Personalausweisgesetz.

In der datenschutzaufsichtsbehördlichen Praxis zeigten sich bei der papierenen Kontaktdatensammlung Probleme, z. B. die Sammlung per Liste, herumfliegende Zettel, in Einzelfällen sogar Missbrauch der Daten für Flirtversuche oder zum Ausforschen der Gäste. Touristen sahen ein Einbruchsrisiko darin, dass sich aus der verpflichtend anzugebenden Postadresse schließen lässt, dass ihre Wohnung oder ihr Haus zurzeit leer steht. Außerdem wurde oft wird die Pflicht zur Information der betroffenen Personen über die Datenverarbeitung nach Art. 13 DSGVO übersehen. ${ }^{9}$

\subsubsection{Sammlung auf Papier}

Viele Kontaktdatensammlungen erfolgen zunächst per ausgelegter Papierliste. Dies steht allerdings nicht im Einklang mit $₫ 4$ Abs. 2 Corona-Bekämpfungsverordnung, da in diesem Fall Unbefugte, also nachfolgende Gäste, Zugriff auf die vorherigen Einträge erlangen. Daher kommen bevorzugt Einzelkontaktdatenformulare zum Einsatz.

Werden sie tagesweise gesammelt, fällt auch das verpflichtende tagesgenaue Löschen leicht, sofern nicht das Gesundheitsamt die Daten wegen eines Infektionsfalls abgefordert hat: Die Papiere, die sich in einem Ordner oder in einer Box befinden, lassen sich ohne weitere Sichtung schreddern.

\subsubsection{Elektronische Datenerhebung}

Auch elektronische Erfassungsmöglichkeiten können die Anforderungen umsetzen, teilweise sogar besser, wenn die Daten nur verschlüsselt hochgeladen gespeichert werden und das Löschen automatisch vollzogen wird. Dennoch gibt es gravierende Nachteile: So ist es schon mehrfach zu Datenlecks der KontaktdatenServer solcher Angebote vorgekommen. Das betrifft dann nicht nur ein einzelnes Restaurant, sondern oft sind in solchen Fällen sämtliche gespeicherte Daten abrufbar. Dann ist das Risiko jedoch viel größer als bei einer dezentralen papierenen Kontaktdatenerhebung, weil nun die Daten leicht verkettet werden können: Aus diesen Daten geht hervor, wer wann wo gegessen hat und wer zur selben Zeit ebenfalls vor Ort war.

Während man einen Verlust des Ordners mit Papier-Kontaktformularen leicht bemerken kann, ist dies nicht unbedingt der Fall bei einem Hacking-Angriff auf einen Webserver mit elektronischen Daten. Hinzu kommt, dass ohnehin nicht jede Person über ein Smartphone verfügt und ihre Daten per App oder Webbrowser melden kann. Das bedeutet für die Gastronomen und Veranstalter, dass ohnehin auch noch eine Lösung ohne Informationstechnik bereitgehalten werden muss.

\subsubsection{Kritik: Rechtsgrundlage}

Bei der Erhebung von Kontaktdaten spielen rechtsstaatliche Erwägungen eine Rolle. So wurde immer wieder kritisiert ${ }^{10,11}$, dass

9 https://www.datenschutzzentrum.de/artikel/1331-Erhebung-von-Kontaktdaten-Informationen-und-Vordrucke.html.

10 Katzenmeier, Grundrechte in Zeiten von Corona, MedR 2020, 461 (462).

11 Pautsch/Haug, Parlamentsvorbehalt und Corona-Verordnung - ein Widerspruch, NJ 2020, 281. die Rechtsgrundlagen der in den Bundesländern getroffenen Maßnahmen in der Regel Rechtsverordnungen sind und daher ein Verstoß gegen den Wesentlichkeitsgrundsatz vorläge.

Zur Erklärung muss dabei zunächst zwischen Gesetzen im formellen und im materiellen Sinne unterschieden werden. Gesetze im formellen Sinne sind nur Parlamentsgesetze, also im Rahmen eines förmlichen Gesetzgebungsverfahren vom Bundes- oder Landtag unter etwaiger Mitwirkung weiterer Gesetzgebungsorgane (etwa Bundesrat) beschlossene und in Gesetzesblättern verkündete Gesetze ${ }^{12}$. Als materielle Gesetze werden dagegen generell-abstrakte Vorschriften bezeichnet, die gegenüber einem Adressatenkreis Rechte und Pflichten begründen, ändern oder aufheben $^{13}$. Durch die Exekutive erlassene Rechtsverordnungen sind daher materielle, aber keine formellen Gesetze. Diese Unterscheidung spielt dann eine Rolle, wenn es auf die Wesentlichkeitstheorie ankommt. Nach dieser vom BVerfG entwickelten und aus dem Rechtsstaats- und Demokratieprinzip abgeleiteten Theorie, müssen „in grundlegenden normativen Bereichen (...) alle wesentlichen Entscheidungen selbst [durch den Gesetzgeber]" ${ }^{\text {"14 }}$ getroffen werden, sie müssen also durch formelle Gesetze geregelt werden.

Mit dieser Frage musste sich nun auch der Verfassungsgerichtshof des Saarlandes beschäftigen ${ }^{15}$. In dem dortigen Verfahren ging es einerseits um die Verpflichtung, bei der Nutzung von öffentlichen Verkehrsmitteln, auf Märkten und in Ladenlokalen eine Mund-Nasen-Bedeckung zu tragen und andererseits um die Datenhebung zum Zwecke der Kontaktnachverfolgung bei Zusammenkünften und Veranstaltungen. Beide Maßnahmen haben ihre Grundlage in der Verordnung zur Bekämpfung der CoronaPandemie ${ }^{16}$. Dabei entschied der Verfassungsgerichthof, dass die Datenerhebung zur Kontaktnachverfolgung mit Art. 2 Satz 2 der Verfassung des Saarlandes unvereinbar sei. Dem Eingriff in das Grundrecht auf Datenschutz (Art. 2 Satz 2 Verfassung des Saarlandes) auf Grundlage der Verordnung fehle es bereits an einer gesetzlichen Ermächtigung. Die Normen des Infektionsschutzgesetzes ( $\$ \$ 28,32$ IfSG) würden als solche nicht ausreichen, da sie für einen generell-abstrakten Eingriff in Art. 2 Satz 2 Verfassung des Saarlandes zu unbestimmt seien. Es bedürfe nach Meinung des Verfassungsgerichtshofes jedenfalls bei Eingriffen, die bereits längere Zeit andauerten und angesichts der konkreten Situation auch voraussichtlich weitere Monate andauern würden, einer parlamentsgesetzlichen Regelung, sodass die Datenerhebung zu diesem Zeitpunkt sowieso nicht mehr auf eine Rechtsverordnung gestützt werden könnte.

\subsubsection{Kritik: Zweckbindung vs. Polizeizugriff}

Zwar ist in $\$ 4$ Abs. 2 der Corona-Bekämpfungsverordnung eine Zweckbeschränkung der erhobenen Daten geregelt. Diese Zweckbeschränkung dürfte jedoch nicht geeignet sein, Anforderungen vonseiten Dritter standzuhalten, die auf einer gesetzlichen Grundlage die Herausgabe verlangen könnten. Ein solches Herausgabeverlangen wäre z. B. eine Beschlagnahme durch Strafverfolgungsbehörden.

Vielmehr bindet die Zweckbeschränkung in erster Linie die erhebenden Stellen selbst. Sie dürfen in eigener Verantwortung die

12 Detterbeck, Allgemeines Verwaltungsrecht, 18. Auflage 2020, § 3 Rn. 88.

13 Detterbeck, a.a.O., § 3 Rn. 89.

14 BVerfG, Beschluss vom 08.08.1978 - 2 BvL 8/77 = BVerfGE 49, 89 (125f).

15 VerfGH des Saarlandes, Beschluss vom 28.08.2020 - Lv 15/20 (in diesem Heft).

16 VO vom 02.05.2020 (Amtsblatt des Saarlandes, Teil II, 22/2020, S. 284f). 
Daten nicht für andere Zwecke verarbeiten. Dies bedeutet nicht nur, dass für eigene andere Zwecke keine Verarbeitung zulässig ist, also z. B. keine Nutzung für Werbezwecke erfolgen darf. Es bedeutet vielmehr auch, dass die speichernde Stelle die Daten auch für fremde andere Zwecke nicht (in eigener Verantwortung) verarbeiten darf. Eine freiwillige Herausgabe an die Polizei auf Grundlage von $\$ 24$ Abs. 1 Nr. 1 BDSG scheidet danach aus. Ihr steht die Zweckbeschränkung der Corona-Bekämpfungsverordnung entgegen.

Anders ist es bei einem Herausgabeverlangen, das die speichernde Stelle (den Gastronomen) zur Herausgabe verpflichtet. Dies kann z. B. ein Beschlagnahmebeschluss sein. In diesem Fall hat der Gastronom nach $\$ 95$ StPO eine rechtliche Verpflichtung zur Herausgabe, die die Anforderungen des Art. 6 Abs. 1 Buchst. c DSGVO erfüllt. Soweit kein Beschlagnahmeschutz nach $\$ 97$ StPO besteht, müssen die Kontaktdaten herausgegeben werden.

Die Beschlagnahme oder das sonstige Herausgabeverlangen muss verhältnismäßig sein. Die Verhältnismäßigkeit bedarf hier einer besonderen Prüfung. Denn auch wenn die Daten (Name, Kontaktdaten, Besuch im Restaurant) für sich genommen oft wenig sensibel sind, muss hier berücksichtigt werden, dass es sich um eine Ausnahmesituation einer Pandemie handelt, die zum Zweck des Infektionsschutzes derartige Kontaktregistrierungen erfordert.

Dieses Verfahren, und damit letztlich auch ein wichtiger Baustein für die Eindämmung der Pandemie, funktioniert nur, wenn alle - Gäste wie auch Gastronomen - daran mitwirken. Hierfür ist das Vertrauen darauf, dass die Angabe der Daten dem eigenen Schutz dient und daraus keine Nachteile entstehen, eine wichtige Grundlage. Dieses Vertrauen genießt daher einen besonderen Schutz. Es hat nicht nur eine individuelle Bedeutung für die Einzelperson, sondern es sollte bei der Verhältnismäßigkeitsprüfung auch der gesamtgesellschaftliche Aspekt beachtet werden. Daher sollten Strafverfolgungsbehörden sorgfältig und nach einem strengen Maßstab prüfen, ob die Beschlagnahme wirklich notwendig und angemessen wäre.

\subsection{Datenschutzfreundlichere Alternativen}

Zum einen könnte man die Datenminimierung vorantreiben: Reicht für das Gesundheitsamt nicht eine Erreichbarkeitsadresse wie z. B. eine Telefonnummer aus, statt dass der Namen und die Postadresse abgefragt werden? Noch besser wäre es, wenn nicht dieselbe Telefonnummer an verschiedenen Stellen hergegeben werden müsste, sondern man wechselnde Pseudonyme verwenden könnte, die einen Kontakt ermöglichen. In der OnlineWelt lassen sich solche Identifikationsdaten kryptografisch generieren; die Online-ID-Funktion des elektronischen Personalausweises könnte hier zum Einsatz kommen. Auch Verfahren wie attributbasierte Berechtigungsnachweise ließen sich verwenden. ${ }^{17}$

Eine prinzipiell funktionierende, wenn auch nicht die schleswig-holsteinische Corona-Bekämpfungsverordnung umsetzende Methode auf Basis von (nicht-wechselnden) Pseudonymen wurde innerhalb von zwei Tagen für Protestaktionen der Klimaschutzbewegung entwickelt: ${ }^{18}$ Hier erhalten die Protestierenden Armbänder mit Pseudonymen aus fünf Buchstaben, die sie bei Kontakten wie einem gemeinsamen Kochen oder Demonstrieren in

17 Rannenberg et al. (Hrsg.), Attribute-based Credentials for Trust. Springer, 2015.

18 Bröckling, Ende Gelände - Aktivist:innen organisieren eigenes Corona-Tracing. Netzpolitik.org, 27.09.2020. https://netzpolitik.org/2020/

ende-gelaende-aktivistinnen-organisieren-eigenes-corona-tracing/\#vorschaltbanner. eine Anwesenheitsliste eintragen. Falls eine Person sich als infiziert meldet, können die anderen Personen, deren Pseudonyme auf denselben Anwesenheitslisten stehen, sich darüber informieren. Allerdings setzt die Methode auf die Eigenverantwortung der Teilnehmenden, selbst wenn aufgrund einer Auflage der Gesundheitsbehörde zusätzlich die Namen und Erreichbarkeitsdaten hinterlegt werden mussten.

\section{Potenziale technischer Lösungen}

Informationstechnik allein kann weder Infektionsrisiken erkennen noch die Pandemie eindämmen: Es gibt beispielsweise keine Sensorik für die Smartphones der Nutzenden, um die Belastung der Umgebungsluft mit Corona-Viren zu messen oder eigenes Biomaterial wie Rachenabstriche auszuwerten. Dennoch kann Technik in Nutzerhand eine Rolle spielen. Neben der CoronaWarn-App ${ }^{19}$, die mit Hilfe von Bluetooth ungefähre Distanzen zwischen Endgeräten (nicht: Personen) feststellt, kommen weitere Apps zum Einsatz, z. B. um in Kombination mit Fitness-Armbändern Messdaten wie Temperatur oder Puls zu analysieren oder als Datenspende zu Forschungszwecken hochzuladen.

Apps können auch in Form anderer Schutzmaßnahmen in der Pandemie-Situation unterstützen, z. B. durch Erinnerung an Hygienemaßnahmen wie das Lüften nach einer definierten Zeit. Auch wenn in der Corona-Pandemie sich eine Schmierinfektion über von mehreren Personen angefasste Gegenstände wie Türklinken als weniger wahrscheinlich herausgestellt hat, können Gebäude künftig so gestaltet werden, dass ein Öffnen von Türen ohne Anfassen möglich ist, beispielsweise über Bewegungssensoren oder im Fall eine Authentifizierungsnotwendigkeit über spezifische Apps.

Allerdings haben sich in den vergangenen Monaten in zahlreichen Apps und angebotenen Diensten - auch für Home Office und Home Schooling - erhebliche Datenschutzdefizite gezeigt. Einerseits fehlt es an einem Selbstverständnis der datenschutzkonformen Systementwicklung, andererseits wurde - spätestens durch die Evaluation zahlreicher Verantwortlicher aufgrund des erneuten „Weckrufs“ des Europäischen Gerichtshofs zum Fall des „Privacy Shield ${ }^{\text {“20 }}$ - deutlich, dass die digitale Souveränität ${ }^{21}$ zu verbessern ist, wenn die Garantie europäischer Grundrechte und Werte auch in technikgestützten Lösungen zum Normalfall werden soll.

\section{5 (Zwischen-)Fazit}

Als Zwischenstand in der aktuellen Pandemie-Situation lässt sich festhalten: Zwar haben Datenschutzargumente bisher eine Dystopie der Vollüberwachung verhindert, aber fast überall muss im Sinne einer Grundrechtskonformität nachgebessert werden - schon deswegen, weil Pandemien mit großer Wahrscheinlichkeit auch noch in vielen Jahren unser Leben begleiten werden.

\footnotetext{
19 Dazu ausführlich Dix, in diesem Heft.

20 EuGH, Urteil vom 16.07.2020 - C-311/18 = DuD 2020, 685.

21 Für den öffentlichen Bereich: DSK, Digitale Souveränität der öffentlichen Verwaltung herstellen - Personenbezogene Daten besser schützen, Entschließung vom 22.09.2020, https://www.datenschutzkonferenz-online.de/media/en/ TOP 8 Entschließung digitale Souveränität_final.pdf.
} 